\title{
Hormonal hippocampus
}

DOI:

10.1038/nrn1917
Growth hormone (GH), which is primarily produced in the pituitary and known for its role in body growth and development, is also expressed in the hippocampus, where its function is not clear. Now, Donahue and coworkers report that the expression of endogenous hippocampal GH in rats is regulated by age, sex and response to stress.

The authors first showed that the level of GH mRNA in the adult hippocampus was more than double that in juveniles. They then compared the levels in adult males and females at different stages of oestrus. Relative to adult males, females in proestrus (a stage associated with ovulation and high blood oestrogen levels) had elevated hippocampal GH mRNA expression, whereas dioestrus females (which had relatively low levels of oestrogen in the blood) did not.

Interestingly, the opposite effect was seen for the growth hormone receptor (GHR) and its downstream effector insulin-like growth factor 1 (IGF1) - expression of both molecules was downregulated by oestrogen. As GH production in the pituitary is normally regulated by negative feedback from IGF1, the authors suggest that the induction of GH mRNA by oestrogen might be due to downregulation of IGF1.

As the production of $\mathrm{GH}$ expression is enhanced by learning, and because stress is known to have different effects on learning in males and females, the authors investigated whether the expression of $\mathrm{GH}$ in the hippocampus is affected by stress. Exposure to brief, intermittent tail shocks increased the expression of GH mRNA in the hippocampus of males and dioestrus females. However, the already high level of GH mRNA expression in the oestrus or proestrus female hippocampus was not affected by stress. Interestingly, the inverse correlation with IGF1 expression was seen in stressed females, but not in stressed males, suggesting that the elevation of GH after stress is regulated by IGF1 in females but by a different mechanism in males.

The sexual dimorphism in hippocampal GH mRNA expression, and the response of GH to stressful experiences, suggest that GH might have a role in hippocampal functions that differ between males and females.

Daniel McGowan

ORIGINAL RESEARCH PAPER Donahue, C. et al. Growth hormone is produced within the hippocampus where it responds to age, sex and stress. Proc. Natl Acad. Sci. USA 103, 6031-6036 (2006)

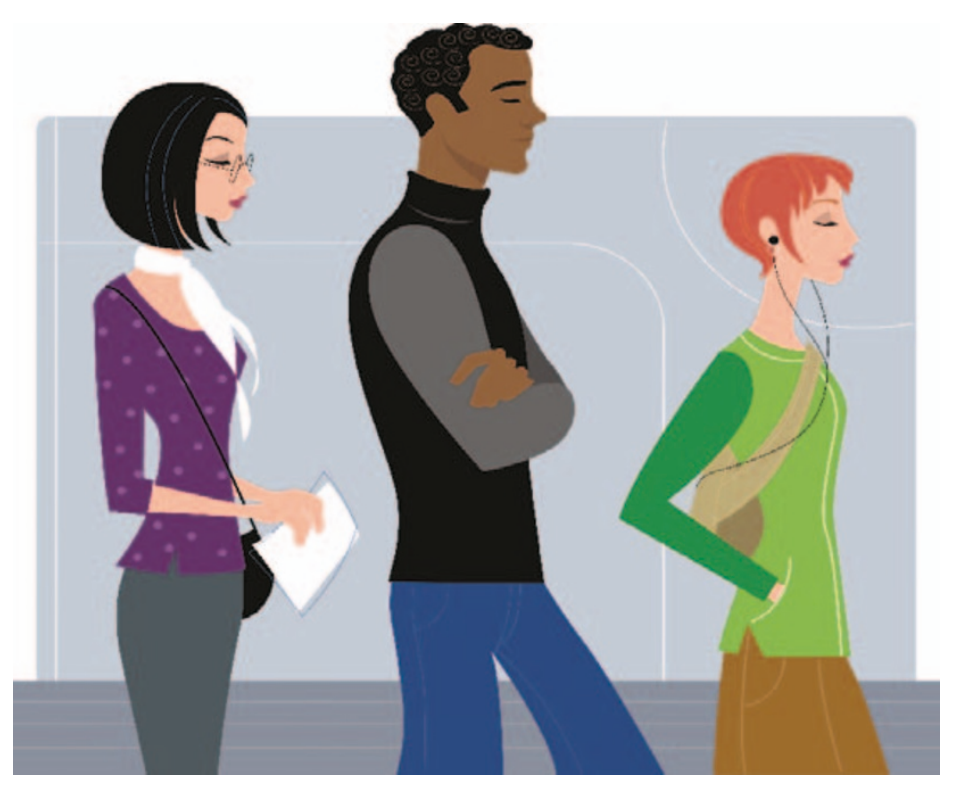

